\title{
Point-by-point model calculation of the prompt neutron multiplicity distribution $v(A)$ in the incident neutron energy range of multi-chance fission
}

\author{
Anabella Tudora ${ }^{1, a}$, Franz-Josef Hambsch ${ }^{2}$, and Viorel Tobosaru ${ }^{1}$ \\ ${ }^{1}$ University of Bucharest, Faculty of Physics, Bucharest Magurele, 405 Atomistilor Str., POB MG-11, 77125, Romania \\ 2 EC-JRC Directorate G - Nuclear Safety and Security, Retieseweg 111, 2440 Geel, Belgium
}

\begin{abstract}
Prompt neutron multiplicity distributions $v(\mathrm{~A})$ are required for prompt emission correction of double energy (2E) measurements of fission fragments to determine pre-neutron fragment properties. The lack of experimental $v(\mathrm{~A})$ data especially at incident neutron energies (En) where the multi-chance fission occurs impose the use of $v(\mathrm{~A})$ predicted by models. The Point-by-Point model of prompt emission is able to provide the individual $v(\mathrm{~A})$ of the compound nuclei of the main and secondary nucleus chains undergoing fission at a given En. The total $v(\mathrm{~A})$ is obtained by averaging these individual $v(\mathrm{~A})$ over the probabilities of fission chances (expressed as total and partial fission cross-section ratios). An indirect validation of the total $v(\mathrm{~A})$ results is proposed. At high En, above $70 \mathrm{MeV}$, the $\mathrm{PbP}$ results of individual $v(\mathrm{~A})$ of the first few nuclei of the main and secondary nucleus chains exhibit an almost linear increase. This shape is explained by the damping of shell effects entering the super-fluid expression of the level density parameters. They tend to approach the asymptotic values for most of the fragments. This fact leads to a smooth and almost linear increase of fragment excitation energy with the mass number that is reflected in a smooth and almost linear behaviour of $v(\mathrm{~A})$.
\end{abstract}

\section{Introduction}

Nowadays there is an interest in the study of neutroninduced fission at intermediate and high energies, justified by the need of nuclear data for both the better understanding of the fission process and new applications, e.g. advanced nuclear systems based on fission, incineration of nuclear waste, isotope production etc. The pre-neutron fragment mass distribution $\mathrm{Y}(\mathrm{A})$ is one of the most important characteristics of the fission process. Even if nuclear modeling has made large progresses in recent years, a pure theoretical description of $\mathrm{Y}(\mathrm{A})$ is far from being achieved. Consequently the fragment mass and kinetic energy distributions $\mathrm{Y}(\mathrm{A}, \mathrm{TKE})$ currently used in the calculation of various quantities characterizing the fission process are experimental data.

Recent experiments regarding fragment distributions of the neutron induced fission of ${ }^{238} \mathrm{U},{ }^{235} \mathrm{U}$ and ${ }^{239} \mathrm{Pu}$ with energies up to $100 \mathrm{MeV}$ were performed. In these experiments the neutron source at LANSCE-WNR providing incident neutron energies (En) from 100's of $\mathrm{keV}$ to 100 's of MeV was used together with a twin Frischgrid ionisation chamber. The double energy (2E) analysis procedure is employed to calculate pre- and post-neutron emission data from fragments.

For such experiments $v(\mathrm{~A})$ distributions are needed in the recovering of pre-neutron fragment masses and distributions from the experimental post-neutron fragment data. It is known that experimental $v(\mathrm{~A})$ data at low En

\footnotetext{
a e-mail: anabellatudora@hotmail.com
}

(e.g. from thermal up to about $5 \mathrm{MeV}$ ) are very scarce, being measured only for a few major actinides. At En above $5-6 \mathrm{MeV}$, where the multi-chance fission occurs, these data are completely missing. The only way is to use $v(\mathrm{~A})$ predicted by models. Consequently the model calculation of $v(\mathrm{~A})$ in the case of multi-chance fission becomes a very important request that the present work addresses.

The present paper focuses on the $v(\mathrm{~A})$ calculation for the fission of ${ }^{238} \mathrm{U}$ and ${ }^{235} \mathrm{U}$ induced by fast neutrons with En ranging from $1 \mathrm{MeV}$ to $80 \mathrm{MeV}$ and from thermal to $20 \mathrm{MeV}$, respectively. The Point-by-Point $(\mathrm{PbP})$ model of prompt emission (described in Ref. [1] and references therein) is used for the first time for multi-chance fission.

$\mathrm{PbP}$ model calculations, including $v(\mathrm{~A})$, are done for each compound nucleus undergoing fission at a given En. The total $v(\mathrm{~A})$ at this En is obtained by averaging the individual $v(\mathrm{~A})$ corresponding to the fissioning compound nuclei over their respective fission chance probabilities. These probabilities are expressed as total and partial fission cross-section ratios RF (for details see Ref. [2] and references therein). At the upper limit of the present calculations, being $\mathrm{En}=80 \mathrm{MeV}$ for $\mathrm{n}+{ }^{238} \mathrm{U}$, the prompt emission from 10 nuclei of the main uranium chain, i.e. ${ }^{239-230} \mathrm{U}$, and 8 nuclei of the protactinium secondary chain, i.e. ${ }^{238-232} \mathrm{~Pa}$, were taken into account.

Because the present $v(\mathrm{~A})$ results, as well as those of other models and procedures used to recover the preneutron fragment data, are predictions (e.g. v(A) provided by the scaling procedure [3] and the GEF code [4], already 
used to obtain the preliminary data reported in Ref. [5]), the possibility of an indirect validation of $v(\mathrm{~A})$ results is proposed, too.

The $v(\mathrm{~A})$ shape and its evolution with increasing En is important on one side for applications and on the other hand for a better understanding of the prompt emission at high En. The evolution of the shapes of individual $v(\mathrm{~A})$ (corresponding to the fissioning compound nuclei involved at the respective En) with increasing En is explained by the influence of shell effects of the fragments. This is reflected in the fragment level density parameters entering the partition of excitation energy at scission. At high excitation energies a damping of shell effects occurs, the level density parameters of a great part of fragments tending to the asymptotic values. This fact leads to an almost smooth and linear increase of the fragment excitation energy with the mass number having as consequence a smooth and almost linear behaviour of individual $v(\mathrm{~A})$.

\section{Features of the modeling}

At En up to about $25 \mathrm{MeV}-30 \mathrm{MeV}$ only the fissioning nuclei of the main chain (formed by neutron emission from the precursor nucleus of this chain) are involved. At En above $30 \mathrm{MeV}$ charged particle emission occurs and the fission of nuclei of secondary chains must be taken into account, too. The prompt emission from nuclei of secondary chains was already included in the extended Los Alamos model (LAM) described in Refs. [1,2]. In the case of $n+{ }^{238} \mathrm{U}$ at En up to $80 \mathrm{MeV}$, the extended LAM calculation of Ref. [2] has taken into account the following nucleus chains and ways: (i) the main nucleus chain, indexed (1) with neutron evaporation from the precursor of this chain; (ii) the "proton" way (denoted as "p") consisting in proton emission from the nuclei of the main chain, leading to nuclei of the secondary chain indexed (2); (iii) the "neutron via proton" (pn) way consisting in neutron evaporation from the precursor of the secondary chain (2); (iv) the "deuteron" way consisting in deuteron emission from the nuclei of the main chain, also leading to nuclei of the secondary chain (2); (v) the "alpha" way consisting in alpha emission from the nuclei of the main chain, leading to the formation of another secondary nucleus chain (3); (vi) the "neutron via alpha" way consisting in neutron evaporation from the precursor of the secondary chain (3). Recursive formulae giving the average excitation energies of the fissioning nuclei formed by the 6 ways mentioned above can be found in Ref. [2].

The plots of total and partial RF reported in Ref. [2] clearly show that RF of the secondary Th chain and the partial RF of the deuteron way are very low (of about $10^{-3}$ and less than $10^{-3}$, respectively). Consequently in this work the contribution of the Th secondary chain and of the "deuteron" way have been neglected.

$\mathrm{PbP}$ calculations are done for the fissioning nuclei of the main $U$ chain in the case of both reactions $n+{ }^{235,238} U$ and for the secondary $\mathrm{Pa}$ chain formed by the ways "proton" and "neutron via proton" in the case of $n+{ }^{238} \mathrm{U}$ at average excitation energies given by the following recursive formulae:

$$
\begin{aligned}
& E x_{1}^{(1)}=E n+B n_{1}^{(1)} \\
& \langle E x\rangle_{i}^{(1)}=\langle E x\rangle_{i-1}^{(1)}-S n_{i-1}^{(1)}-\left\langle\varepsilon_{e v}\right\rangle_{i-1}^{(1)}, \quad i=2, \ldots N^{(1)}
\end{aligned}
$$

in which " $i$ " denotes the fission chance, $N^{(1)}$ is the number of nuclei of the main chain, $B n^{(1)} / S n^{(1)}$ are the neutron binding/separation energies and $\left\langle\varepsilon_{e v}\right\rangle^{(1)}$ are the average energies of the neutrons evaporated before fission (prefission neutrons) in the center-of-mass system.

$$
\begin{aligned}
\langle E x\rangle_{i}^{(p)} & =\langle E x\rangle_{i}^{(1)}-S_{p i}^{(1)}-\left\langle\varepsilon_{e v}\right\rangle_{p i}, i=1, \ldots, N^{(2)} \\
\langle E x\rangle_{i}^{(p n)} & =\langle E x\rangle_{i-1}^{(p n)}-S n_{i-1}^{(2)}-\left\langle\varepsilon_{e v}\right\rangle_{n, i-1}^{(2)}, i=2, \ldots, N^{(2)} \\
\langle E x\rangle_{1}^{(p n)} & =\langle E x\rangle_{1}^{(p)}
\end{aligned}
$$

In Eqs. $(2,3) N^{(2)}$ is the number of nuclei of the secondary chain (2), $S p^{(1)}$ are the proton separation energies from the nuclei of the main chain, $S n^{(2)}$ are the neutron separation energies from the nuclei of the chain $(2),\left\langle\varepsilon_{e v}\right\rangle_{p}$ and $\left\langle\varepsilon_{e v}\right\rangle_{n}^{(2)}$ are the average energies of the evaporated proton and neutron before fission, respectively.

For all $\mathrm{U}$ and $\mathrm{Pa}$ nuclei the fragmentation range taken in the $\mathrm{PbP}$ calculations was constructed as usual (see Ref. [1] for details) i.e. for each mass number A covering a large range from symmetric fission up to a very asymmetric split, three charge numbers $\mathrm{Z}$ are taken as the nearest integer values above and below the most probable charge $\mathrm{Zp}(\mathrm{A})$ taken as $\mathrm{Z}_{U C D}(\mathrm{~A})$ corrected with the charge polarization $\Delta \mathrm{Z}(\mathrm{A})$ (which is taken here as $|0.5|$ for all fragmentations, with plus sign for the light fragments and minus sign for the complementary heavy ones). The isobaric charge distributions are taken as Gaussian functions centred on $\mathrm{Zp}(\mathrm{A})$ with the same root-meansquare value of 0.6 for all fragmentations. The features of the $\mathrm{PbP}$ model were described in previous publications (see Ref. [1] and references therein). Here we mention only that the compound nucleus cross-sections of the inverse process of neutron evaporation from all fragments coming from $\mathrm{U}$ and $\mathrm{Pa}$ nuclei are provided by optical model calculations with the phenomenological potential of Becchetti-Greenlees. The level density parameters of fragments are calculated using the super-fluid model two times, first at scission in the frame of the excitation energy partition (implying an iterative procedure under the condition of statistical equilibrium at scission) and second at full acceleration.

The $\mathrm{PbP}$ results of prompt neutron multiplicity of fragments corresponding to each fissioning compound nucleus are averaged over the isobaric charge distributions, giving the individual prompt neutron multiplicity distribution $v_{i}(\mathrm{~A})$. The total $v(\mathrm{~A})$ at a given En is then obtained by averaging these individual $v_{i}(\mathrm{~A})$ over the probabilities of fission chances (expressed by total and partial RF):

$$
v(A)=\sum_{k=1}^{3} \sum_{i=1}^{N(k)} R F_{i}^{(k)} v_{i}^{(k)}(A)
$$

in which $\mathrm{k}$ denotes the fission ways, i.e. the three ones, (i)-(iii) taken into account for $n+{ }^{238} \mathrm{U}$ and only the main way in the case of $n+{ }^{235} \mathrm{U}$. A similar formula can be written also for the prompt neutron multiplicity of a fragmentation (fragment pair). The average total number of prompt neutrons emitted by the fission fragments $<v_{\mathrm{FF}}$ can be obtained by averaging $v(\mathrm{~A})$ of Eq. (4) over any $\mathrm{Y}(\mathrm{A})$ distribution, if available:

$$
<v>_{\mathrm{FF}}=\sum_{A} v(A) Y(A) / \sum_{A} Y(A)
$$




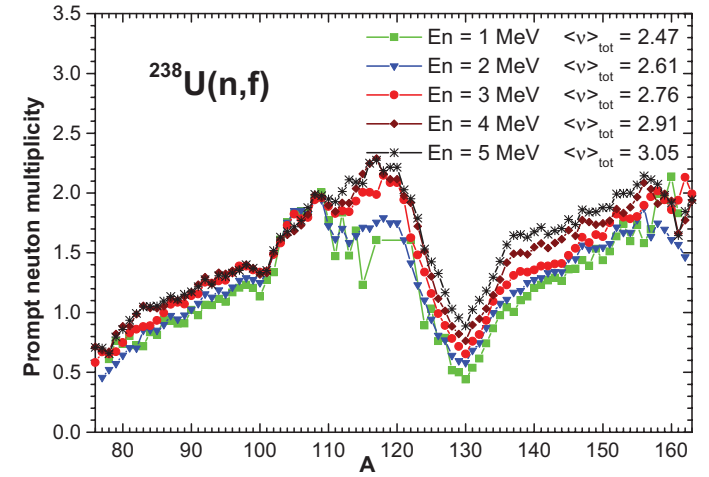

Figure 1. $v(\mathrm{~A})$ of ${ }^{238} \mathrm{U}(\mathrm{n}, \mathrm{f})$ at En from $1 \mathrm{MeV}$ to $5 \mathrm{MeV}$.

At En where only one fission chance is involved $\langle\nu\rangle_{F F}$ is just the total average number of prompt neutrons $\langle\nu\rangle_{\text {tot }}$ which is experimentally measured. At higher En where the multi-chance fission occurs, $\langle v\rangle_{\text {tot }}$ is the sum of $\langle v\rangle_{F F}$ emitted by fission fragments and the total average number of neutrons evaporated from the compound nuclei before fission, usually named pre-fission neutrons $\langle v\rangle_{\text {prefiss: }}$ :

$$
<v>_{\text {tot }}=<v>_{F F}+<v>_{\text {prefiss }}
$$

$\langle v\rangle_{\text {prefiss }}$ depends on the probability of nuclei formed by neutron evaporation from the precursor of the main chain and from the secondary chains by the ways "neutron via proton" and "neutron via alpha". It can be easily calculated as:

$$
<v>_{\text {prefiss }}=\sum_{k=1,3,6} \sum_{i=1}^{N(k)}(i-1) R F_{i}^{(k)}
$$

Taking into account the scarcity of experimental $v(\mathrm{~A})$ data and the fact that $\langle v\rangle_{\text {tot }}$ as a function of En is the only prompt neutron data measured in a sufficient amount, the relations (5)-(7) can serve for an indirect validation of the calculated $v(\mathrm{~A})$ by comparing the obtained $\langle v\rangle_{\text {tot }}$ with these experimental data.

\section{PbP model calculations of $v(\mathrm{~A})$}

\section{1. $\mathrm{PbP}$ results of $v(\mathrm{~A})$ below the threshold of the second-chance fission}

Examples of $\mathrm{PbP}$ model results of $v(\mathrm{~A})$ in the En range where only the first-chance fission is involved are given in Fig. 1 for ${ }^{238} \mathrm{U}(\mathrm{n}, \mathrm{f})$.

The increase of the prompt neutron multiplicity with increasing En mainly for heavy fragments is easily seen. In the case of ${ }^{235} \mathrm{U}(\mathrm{n}, \mathrm{f})$ experimental $v(\mathrm{~A})$ data exist at thermal En and at $\mathrm{En}=0.5 \mathrm{MeV}$ and $5.5 \mathrm{MeV}$.

$\mathrm{PbP}$ results of $v(\mathrm{~A})$ describing very well these experimental data were already reported in Refs. $[1,6,7]$ and references therein.

\subsubsection{Demonstration of the possibility of an indirect validation of the $v(A)$ results}

The indirect validation of the $v(\mathrm{~A})$ results implies the calculation of $\langle v\rangle_{\mathrm{FF}}$ according to Eq. (5) and the comparison of $\langle v\rangle_{\text {tot }}$ with the existing experimental data. This validation is based on the fact that the $v(\mathrm{~A})$ distribution has a strong influence on the $\langle\nu\rangle_{\text {FF }}$ value

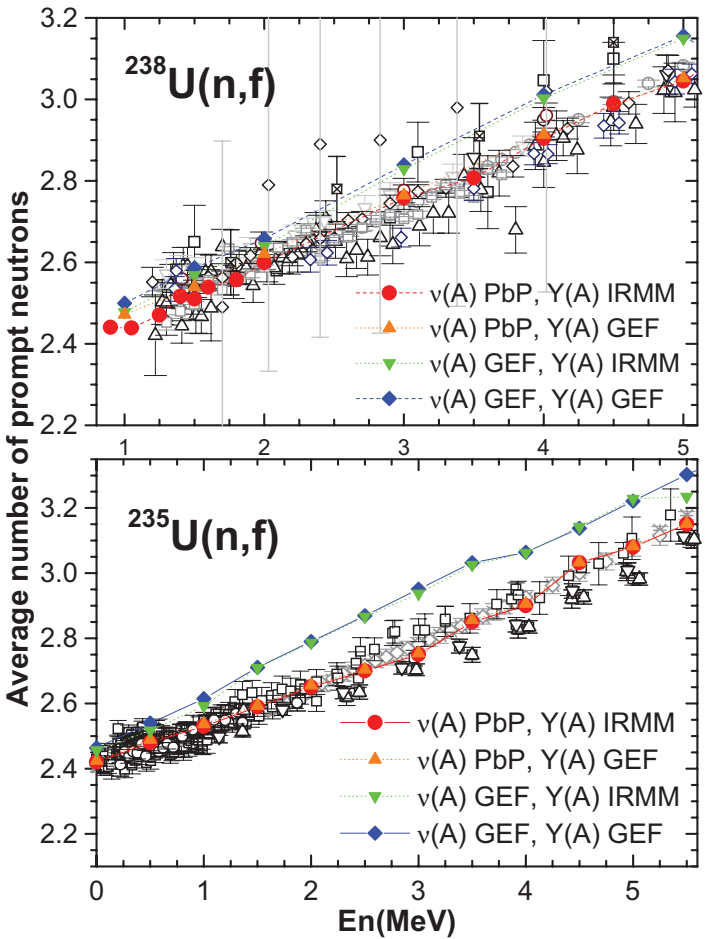

Figure 2. $\langle v\rangle_{\text {tot }}$ of ${ }^{238} \mathrm{U}(\mathrm{n}, \mathrm{f})$ (upper part) and ${ }^{235} \mathrm{U}(\mathrm{n}, \mathrm{f})$ (lower part) obtained by averaging different $v(\mathrm{~A})$ over different $\mathrm{Y}(\mathrm{A})$

while the $\mathrm{Y}(\mathrm{A})$ distribution has only a weak influence. A simple exercise (illustrated in Fig. 2), consisting in the comparison of $\langle\nu\rangle_{F F}$ obtained by averaging a given $v(\mathrm{~A})$ distribution over different $\mathrm{Y}(\mathrm{A})$ distributions clearly demonstrates this fact. The $\mathrm{PbP}$ results of $v(\mathrm{~A})$ of ${ }^{238,235} \mathrm{U}(\mathrm{n}, \mathrm{f})$ are averaged over the experimental $\mathrm{Y}(\mathrm{A})$ data of JRC-IRMM [8-10] (red circles) and over the Y(A) distributions provided by the GEF code [4] (orange up triangles). Another $v(\mathrm{~A})$ distribution, this time provided by the GEF code [4], was also averaged over both $\mathrm{Y}(\mathrm{A})$ distributions mentioned above (green down triangles and blue diamonds).

As it can be seen for both studied fissioning systems, the $v(\mathrm{~A})$ distribution of $\mathrm{PbP}$ averaged over $\mathrm{Y}(\mathrm{A})$ of JRCIRMM and GEF gives close results (red circles compared with orange up triangles). The $v(\mathrm{~A})$ of GEF averaged over these $\mathrm{Y}(\mathrm{A})$ gives close results, too (green down triangles compared with blue diamonds). The overestimation of experimental data (different black and grey symbols) by the $\langle v\rangle_{\text {tot }}$ results based on $v(\mathrm{~A})$ of GEF, visible for both fissioning systems, is due to the $v(\mathrm{~A})$ distributions of GEF that are higher than those of PbP. It can be also seen that $\langle v\rangle_{\text {tot }}$ based on the $v(\mathrm{~A})$ distributions of $\mathrm{PbP}$ (averaged over two different $\mathrm{Y}(\mathrm{A})$ distributions) describes very well the experimental data of both fissioning systems ${ }^{238,235} \mathrm{U}(\mathrm{n}, \mathrm{f})$.

\section{2. $\mathrm{PbP}$ results of $v(\mathrm{~A})$ at En where the multi-chance fission occurs}

An example of $v(A)$ results is given in Fig. 3 for $n+{ }^{238} U$ at $\mathrm{En}=80 \mathrm{MeV}$. The individual $v_{i}(\mathrm{~A})$ of the nuclei of the main chain ${ }^{239-230} \mathrm{U}$ are plotted with different open symbols and those of the secondary nuclei ${ }^{238-232} \mathrm{~Pa}$ with continuous lines. The total $v(\mathrm{~A})$ obtained according to Eq. (4) (in which the RF of the BRC evaluation [11] were 


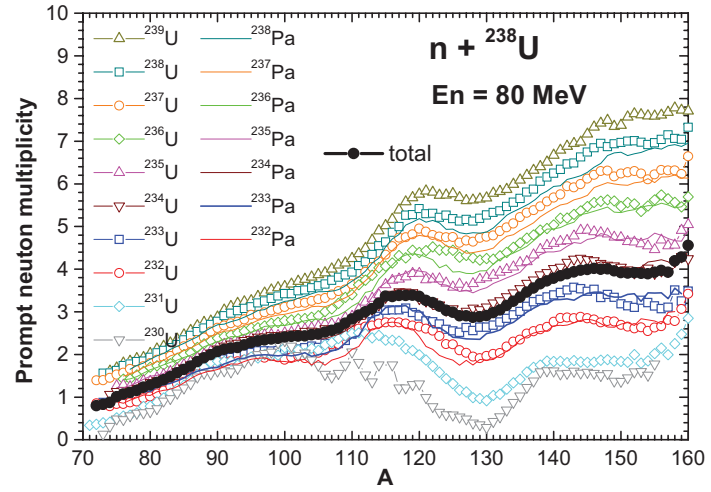

Figure 3. $\mathrm{PbP}$ results of individual $v(\mathrm{~A})$ (open symbols and lines) and total $v(\mathrm{~A})$ (full black circles) of $\mathrm{n}+{ }^{238} \mathrm{U}$ at $\mathrm{En}=80 \mathrm{MeV}$.

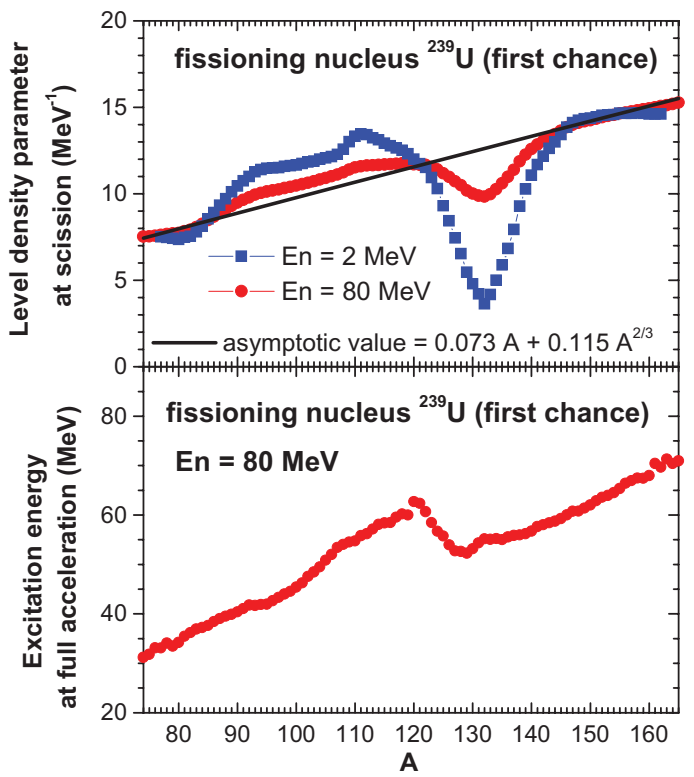

Figure 4. Upper part: fragment level density parameters at scission of the main fissioning nucleus ${ }^{239} \mathrm{U}$ at En $=80 \mathrm{MeV}$ (red circles) and $2 \mathrm{MeV}$ (blue squares). Lower part: fragment excitation energy at full acceleration for the main compound nucleus ${ }^{239} \mathrm{U}$ at $\mathrm{En}=80 \mathrm{MeV}$.

used) is plotted with full black circles. As it can be seen the sawtooth shape of individual $v(\mathrm{~A})$ corresponding to the first few compound nuclei of the $\mathrm{U}$ and $\mathrm{Pa}$ chains (having the highest excitation energies) is almost vanished.

The explanation is the damping of shell effects of most fragments coming from these nuclei. The available excitation energy at scission of many fragmentations is high enough to reach the asymptotic values of the fragment level density parameters entering the energy partition. This is exemplified in the upper part of Fig. 4 showing the level density parameters of fragments at scission for the main compound nucleus ${ }^{239} \mathrm{U}$ at a high En of $80 \mathrm{MeV}$ (red circles) in comparison with a low En of $2 \mathrm{MeV}$ (blue squares). As it can be seen at $\mathrm{En}=80 \mathrm{MeV}$ the level density parameters of a great part of fragments (except around A of 132) are close to the asymptotic value plotted with a solid line. The behaviour of the level density parameters at high excitation energies (due to the damping of shell effects) is reflected in an almost linear increase of the individual fragment excitation energy $\mathrm{E}^{*}(\mathrm{~A})$ at full acceleration, visible in the lower part of Fig. 4, and as consequence in the almost linear shape of individual $v(\mathrm{~A})$.

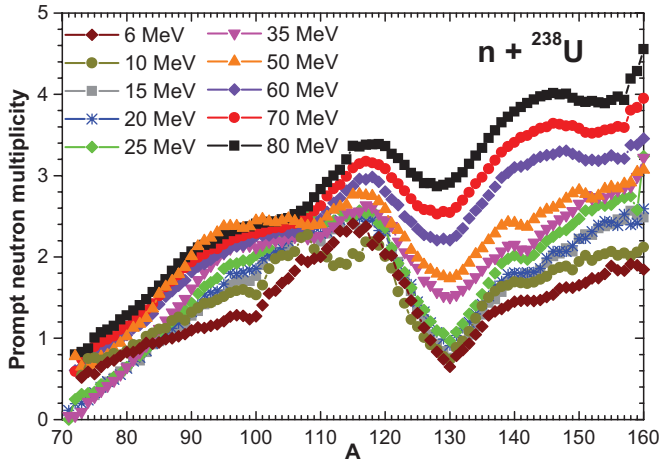

Figure 5. $\mathrm{PbP}$ results of total $v(\mathrm{~A})$ of $\mathrm{n}+{ }^{238} \mathrm{U}$ at ten En ranging from $6 \mathrm{MeV}$ to $80 \mathrm{MeV}$.

Examples of total $v(A)$ of the reaction $n+{ }^{238} \mathrm{U}$ calculated at ten incident energies ranging from $6 \mathrm{MeV}$ to $80 \mathrm{MeV}$ are plotted together in Fig. 5. An almost washing out of the sawtooth shape of total $v(\mathrm{~A})$ with increasing En is visible, too.

In order to see the impact of different RF on the total $v(\mathrm{~A})$ obtained according to Eq. (4), the PbP results of individual $v_{i}(\mathrm{~A})$ were also averaged over other fission cross-section ratios, e.g. those from the ENDF/B-VII [12] and JENDL4 [13] evaluations in the case of ${ }^{238} \mathrm{U}$. The differences versus the total $v(\mathrm{~A})$ obtained with RF of BRC are almost insignificant.

The $v(\mathrm{~A})$ results of $\mathrm{PbP}$ were also compared with those provided by the GEF code [4] and the scaling method [3] which were already used to provide the preliminary pre-neutron fragment distributions of $n+{ }^{238} \mathrm{U}$ up to $\mathrm{En}=20 \mathrm{MeV}$ reported in Ref. [5]. Examples of such a comparison are given in Fig. 6 for $n+{ }^{238} \mathrm{U}$ at $\mathrm{En}=20 \mathrm{MeV}$ and $80 \mathrm{MeV}$. The $v(\mathrm{~A})$ results of the GEF code versions 2014/2.1 and 2015/2.2 [4] are plotted with full blue circles and open red circles, respectively, the scaling method result is given with a dark-yellow line and the $\mathrm{PbP}$ results with black stars.

Differences in shape between the $v(\mathrm{~A})$ results of the versions of GEF are visible, especially at A between 120 and 135. The $v(\mathrm{~A})$ results of $\mathrm{PbP}$ are lower than those of GEF and exhibit a different shape compared to the GEF results. $v(\mathrm{~A})$ of the scaling method differ very much from the $\mathrm{PbP}$ and GEF results, exhibiting a much more pronounced sawtooth shape. At high En values, e.g. $80 \mathrm{MeV}$, the $v(\mathrm{~A})$ result of $\mathrm{PbP}$ has a visible tendency to a linear increase, exhibiting only a slight oscillation in the A region of symmetric fission compared to the line plotted in wine color. The $v(\mathrm{~A})$ results of both GEF versions show more pronounced deviations from linearity.

The differences between the $v(\mathrm{~A})$ results of $\mathrm{PbP}$ and GEF can be understood by the differences of principle existing between the TXE partition methods of $\mathrm{PbP}$ and GEF. These methods were analysed in detail in Ref. [6].

\subsubsection{Comparison of $\langle\boldsymbol{v}\rangle_{\text {tot }}$ with experimental data and indirect validation of $v(A)$}

The very low influence of the $\mathrm{Y}(\mathrm{A})$ distribution on $\langle v\rangle_{\mathrm{FF}}$ given by Eq. (5), as demonstrated in Sect. 3.1.1, allows the indirect validation of the $v(\mathrm{~A})$ results by comparing $\langle v\rangle_{\text {tot }}$ given by Eq. (6) with the existing experimental data.

Available $\mathrm{Y}(\mathrm{A})$ distributions at En where the multichance fission occurs, are those provided by the GEF code 


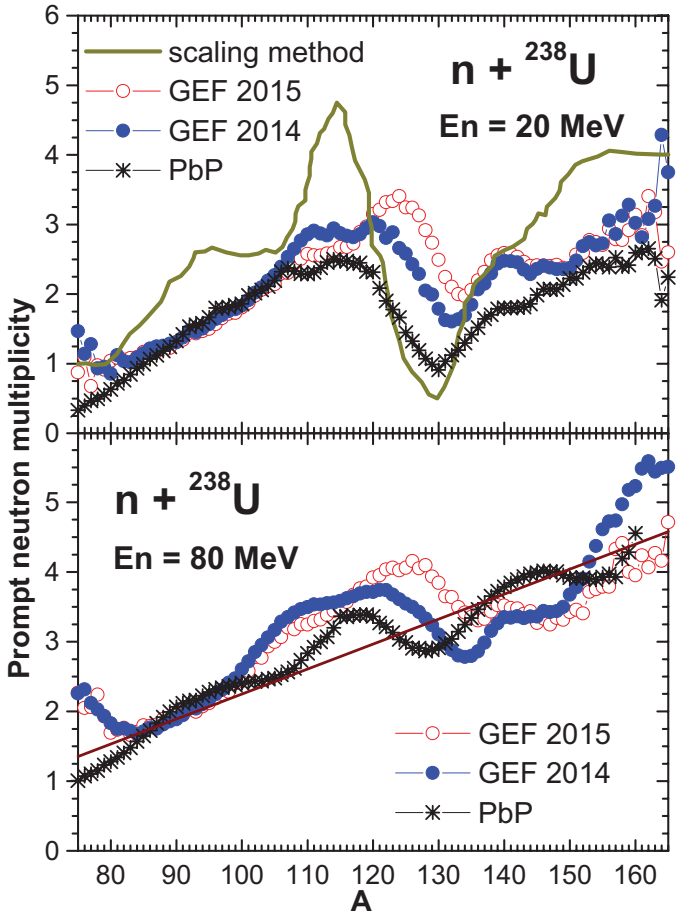

Figure 6. Comparison between the $v(\mathrm{~A})$ results of $\mathrm{PbP}, \mathrm{GEF}$ and scaling method for the case of $n+{ }^{238} \mathrm{U}$ at two En.

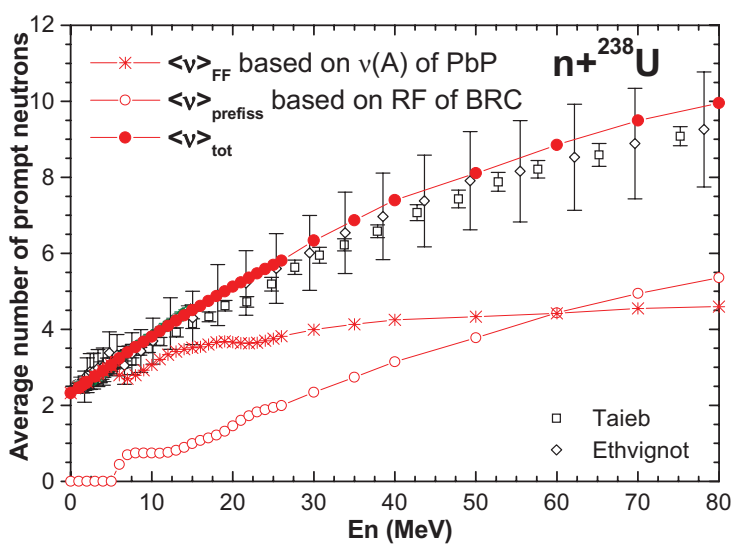

Figure 7. $\left\langle v>_{\mathrm{FF}}\right.$ (red stars), $\langle v\rangle_{\text {prefiss }}$ (open red circles) and $\langle v\rangle_{\text {tot }}$ (full red circles) of $\mathrm{n}+{ }^{238} \mathrm{U}$ in comparison with experimental data (open black symbols).

[4]. $\langle v\rangle_{\mathrm{FF}}$ obtained by averaging $v(\mathrm{~A})$ of $\mathrm{PbP}$ over $\mathrm{Y}(\mathrm{A})$ of GEF, are plotted with red stars in Fig. 7 referring to $\mathrm{n}+{ }^{238} \mathrm{U}$ up to $\mathrm{En}=80 \mathrm{MeV}$ and Fig. 8 referring to $\mathrm{n}+{ }^{235} \mathrm{U}$ up to $\mathrm{En}=20 \mathrm{MeV}$.

The average number of pre-fission neutrons $\langle v\rangle_{\text {prefiss }}$ calculated according to Eq. (7) are plotted in Figs. 7 and 8 with open red circles. They are based on RF of the BRC evaluation [11] in the case of $n+{ }^{238} U$ and RF of the ENDF/B-VII.1 evaluation [14] for $\mathrm{n}+{ }^{235} \mathrm{U}$. The resulting $\langle\nu\rangle_{\text {tot }}$ is plotted with full red circles in both figures.

As it can be seen, $\langle v\rangle_{\text {tot }}$ calculated for both fissioning systems reproduce the experimental data.

For comparison in Fig. 8 the results provided by the GEF code [4] are plotted using the same symbols colored in blue. $\langle v\rangle_{\text {tot }}$ of GEF slightly overestimates the experimental data at En from thermal up to $15 \mathrm{MeV}$. Because $\langle v\rangle_{\text {prefiss }}$ of GEF (open blue circles) is close but lower than $\langle v\rangle_{\text {prefiss }}$ used in the present calculations

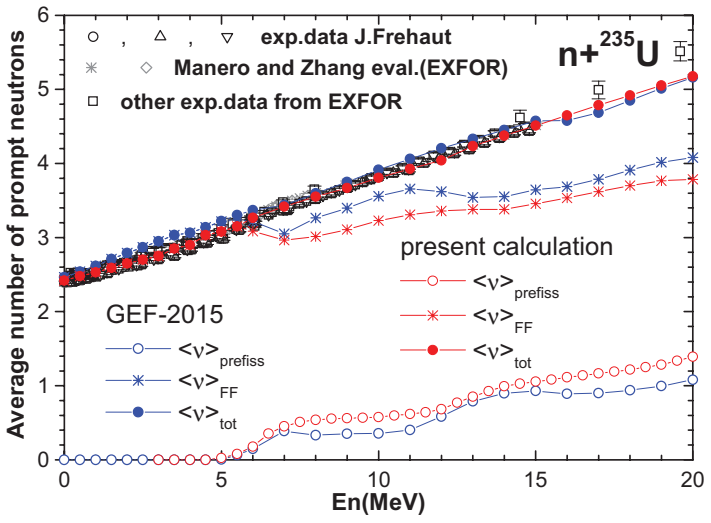

Figure 8. $\langle v\rangle_{\mathrm{FF}}$ (stars), $\langle v\rangle_{\text {prefiss }}$ (open circles) and $\langle v\rangle_{\text {tot }}$ (full circles) of $n+{ }^{235} \mathrm{U}$, in comparison with experimental data (different open black and gray symbols. The present results are plotted in red color and those of the GEF code in blue color.

(given with open red circles) it is obvious that the overestimation of experimental data by the GEF results is exclusively due to the higher values of $\langle v\rangle_{\mathrm{FF}}$, i.e. to the higher $v(\mathrm{~A})$ provided by the GEF code.

It can be also seen that at $\mathrm{En}=16 \mathrm{MeV}$ the $\langle v\rangle_{\text {tot }}$ value of GEF is almost the same as at $15 \mathrm{MeV}$, leading at En above $15 \mathrm{MeV}$ to a lower $\langle v\rangle_{\text {tot }}$ result than the one of $\mathrm{PbP}$.

\section{Conclusions}

Post-neutron fragment data for the reactions $\mathrm{n}+{ }^{238,235} \mathrm{U}$ at En up to several tens of $\mathrm{MeV}$ were measured in a recent experiment at the LANSCE facility. To obtain pre-neutron fragment distributions these data need the correction for prompt neutron emission. Consequently $v(\mathrm{~A})$ distributions are required. The lack of experimental $v(\mathrm{~A})$ data, at En where the multi-chance fission occurs, impose the use of $v(\mathrm{~A})$ predicted by models.

The $\mathrm{PbP}$ model of prompt emission is able to provide the individual $v(\mathrm{~A})$ of compound nuclei of the main and secondary nucleus chains that are undergoing fission at a given En. The total $v(\mathrm{~A})$ is then obtained by averaging these individual $v(\mathrm{~A})$ distributions over the fission chance probabilities expressed as total and partial fission cross section ratios.

The $\mathrm{PbP}$ calculations showed that the sawtooth shape of individual $v(\mathrm{~A})$ becomes less pronounced with increasing excitation energy of the respective compound nucleus undergoing fission. At high En, e.g. $70 \mathrm{MeV}$, $80 \mathrm{MeV}, v(\mathrm{~A})$ of the first few fissioning nuclei of the $\mathrm{U}$ and Pa chains acting in the reaction $n+{ }^{238} \mathrm{U}$, having the highest excitation energies, exhibit an almost linear increase. This shape is explained by the complete damping of shell effects in the super-fluid expression of the level density parameter of a great part of fragments. This fact leads to a smooth and almost linear increase of the fragment excitation energy with the mass number $\mathrm{A}$ that is reflected in a smooth and almost linear behaviour of individual $v(\mathrm{~A})$ distribution.

An indirect validation of $v(\mathrm{~A})$ is possible through the comparison of the calculated $\langle v\rangle_{\text {tot }}$ with existing experimental data. This comparison can be used as an indirect validation of predicted $v(\mathrm{~A})$ because the magnitude of $\langle v\rangle_{\mathrm{FF}}$ (obtained by averaging $v(\mathrm{~A})$ over $\mathrm{Y}(\mathrm{A}))$ is strongly dependent on the $v(\mathrm{~A})$ distribution and has only a weak dependence on the $\mathrm{Y}(\mathrm{A})$ distribution. The $\mathrm{PbP}$ results of $v(\mathrm{~A})$ averaged over available $\mathrm{Y}(\mathrm{A})$ 
distributions (i.e. experimental Y(A) of IRMM and Y(A) provided by the GEF code) lead to $\langle\nu\rangle_{\text {tot }}$ in good agreement with the experimental data.

The differences between the total $v(\mathrm{~A})$ obtained by averaging the $\mathrm{PbP}$ results of individual $v(\mathrm{~A})$ over $\mathrm{RF}$ of different evaluations (e.g. ENDF/B-VII.1, JENDL4, JEFF3.2) are almost insignificant.

The comparison of the present results with those of the GEF code, justified by the use of $v(\mathrm{~A})$ predicted by GEF to obtain the preliminary pre-neutron fragment distributions of the reactions $n+{ }^{238,235} \mathrm{U}$ up to $\mathrm{En}=20 \mathrm{MeV}$ reported in Ref. [5], revealed significant differences between the $v(\mathrm{~A})$ results of $\mathrm{PbP}$ and GEF. Especially the $v(\mathrm{~A})$ shapes at high En are different. In the case of $n+{ }^{238} \mathrm{U}$ the $\mathrm{PbP}$ results of $\nu(\mathrm{A})$ reflect the influence of the almost linear shape of individual $v(\mathrm{~A})$ corresponding to the first few fissioning nuclei of the $\mathrm{U}$ and $\mathrm{Pa}$ chains. The $v(\mathrm{~A})$ results of GEF at high En are deviating from this linear tendency.

The significant differences in shape and magnitude between the $v(\mathrm{~A})$ predictions of $\mathrm{PbP}$ and $\mathrm{GEF}$ for both reactions $n+{ }^{238} U$ and $n+{ }^{235} U$ are expected to influence the pre-neutron fragment distributions obtained from the postneutron fragment data, that will be the subject of a future paper.

\section{References}

[1] R. Capote, Chen Y.J., F.-J. Hambsch, N. Kornilov, J.P. Lestone, O. Litaize, B. Morillon, D. Neudecker, S. Oberstedt, N. Otuka, V.G. Pronyaev, A. Saxena, O. Serot, O.A. Scherbakov, Shu N.C., D.L. Smith, P. Talou, A. Trkov, A.C. Tudora, R. Vogt and A.S. Vorobyev, Nucl. Data Sheets 131, 1 (2016)
[2] A. Tudora, G. Vladuca, B. Morillon, Nucl. Phys. A 740, 33 (2004)

[3] J.P. Lestone, Nucl. Data Sheets 112, 3120 (2011)

[4] K.-H. Schmidt and B. Jurado, GEF code versions 2014/2.1 and 2015/2.2, available online: http://www.khs-erzhausen.de/GEF.html

[5] F.-J. Hambsch, A. Tudora, D.L. Duke, F. Tovesson, In. Conf. PHYSOR "Unified theory and experiments in the 21-st century”, May 1-5, 2016, Sun Valley, Idaho, USA

[6] A. Tudora, F.-J. Hambsch, I. Visan, G. Giubega, Nucl. Phys. A 940, 242 (2015)

[7] A. Tudora, F.-J. Hambsch, S. Oberstedt, G. Giubega, I. Visan, Nucl. Sci. Eng. 181, 289 (2015)

[8] E. Birgersson, A. Oberstedt, S. Oberstedt, F.-J. Hambsch, Nucl. Phys. A 817, 1 (2009)

[9] F. Vivès, F.-J. Hambsch, H. Bax, S. Oberstedt. Nucl. Phys. A 662, 63 (2000)

[10] Ch. Straede, C. Budtz-Jorgensen and H.H. Knitter, Nucl. Phys. A 462, 85 (1987)

[11] Evaluated Nuclear Data File JEFF3.2 (2014), original data taken from JEFF3.1.1 (CEA/DAM), files $\mathrm{ZA}=092238, \mathrm{MF}=3, \mathrm{MT}=18,19,20,21,38$. Available online https://www-nds . iaea.org

[12] Evaluated Nuclear Data File ENDF/B-VII.1 (2011), files $\mathrm{ZA}=092238, \mathrm{MF}=3, \mathrm{MT}=18,19,20,21$, 38. Available online https ://www-nds . iaea.org

[13] Evaluated Nuclear Data File JENDL4 (2012), files $\mathrm{ZA}=092238, \mathrm{MF}=3, \mathrm{MT}=18,19,20,21,38$. Available online https://www-nds . iaea.org

[14] Evaluated Nuclear Data File ENDF/B-VII.1 (2011), files $\mathrm{ZA}=092235, \mathrm{MF}=3, \mathrm{MT}=18,19,20,21$, 38. Available online https : //www-nds . iaea.org 